\begin{tabular}{|c|c|}
\hline Title & Carrier pocket engineering to design superior thermoelectric materials using GaA s/A IA s superlattices \\
\hline Author(s) & Koga, T.; Sun, X.; Cronin, S. B.; Dressel haus, M. S. \\
\hline Citation & $\begin{array}{l}\text { A pplied Physics Letters, } 73(20), 2950-2952 \\
\text { https://doi.org/10.1063/1.122640 }\end{array}$ \\
\hline Issue Date & $1998-11-16$ \\
\hline Doc URL & http:/hdl.handle.net/2115/45770 \\
\hline Rights & $\begin{array}{l}\text { Copyright } 1998 \text { A merican Institute of Phy sics. This article may be downloaded for personal use only. A ny other use } \\
\text { requires prior permission of the author and the A merican Institute of Physics. The following article appeared in A ppl. } \\
\text { Phys. Lett. } 73,2950 \text { (1998) and may be found at https://dx.doi.org/10.1063/1.122640 }\end{array}$ \\
\hline Type & article \\
\hline File Information & APL73_2950.pdf \\
\hline
\end{tabular}

Instructions for use 


\title{
Carrier pocket engineering to design superior thermoelectric materials using GaAs/AIAs superlattices
}

\author{
T. Koga, ${ }^{\text {a) }}$ X. Sun, S. B. Cronin, and M. S. Dresselhaus ${ }^{\text {b) }}$ \\ Department of Physics, Massachusetts Institute of Technology, Cambridge, Massachusetts 02139
}

(Received 9 July 1998; accepted for publication 17 September 1998)

\begin{abstract}
A large enhancement in the thermoelectric figure of merit for the whole superlattice, $Z_{3 \mathrm{D}} T$, is predicted for short-period GaAs/AlAs superlattices relative to bulk GaAs. Various superlattice parameters (superlattice growth direction, superlattice period, and layer thicknesses) are explored to optimize $Z_{3 \mathrm{D}} T$, including quantum well states formed from carrier pockets at various high symmetry points in the Brillouin zone. The highest room-temperature $Z_{3 \mathrm{D}} T$ obtained in the present calculation is 0.41 at the optimum carrier concentration for either (001)- or (111)-oriented GaAs $(20 \AA)$ / AlAs (20 ̊) superlattices, which is about 50 times greater than the corresponding $Z T$ for bulk GaAs. (C) 1998 American Institute of Physics. [S0003-6951(98)01546-0]
\end{abstract}

There have been an increasing number of studies on the enhanced thermoelectric figure of merit $Z\left(=S^{2} \sigma / \kappa\right)$, where $S$ is the Seebeck coefficient, $\sigma$ is the electrical conductivity, and $\kappa$ is the thermal conductivity, for a material in the form of a multiple-quantum-well (MQW) structure. ${ }^{1-15}$ The most popular strategy in this research area is to take advantage of the enhanced density of states for electrons near the Fermi level due to the reduced dimensionality. ${ }^{1-12}$ In this approach, in-plane thermoelectric transport of the MQW sample should be enhanced due to the (1) enhanced Seebeck coefficient relative to the bulk value at a given carrier concentration, (2) enhanced electron carrier mobility due to the $\delta$ - and modulation-doping techniques, and (3) reduced lattice thermal conductivity due to the boundary scattering of phonons at the barrier-well interfaces. It is, however, noted that although a fairly large enhancement (by a factor of several) in $Z T$ is expected within the quantum well, ${ }^{1-5}$ there has been little work on predicting a large enhancement in $Z T$ for the whole superlattice.

In this letter, we first show that the quantum-well approach is indeed effective in enhancing $Z T$ for the whole superlattice using a GaAs/AlAs $\Gamma$-point quantum-well superlattice, where we assume that only the $\Gamma$-point subband is occupied by electrons. Second, we show the possibility of another degree of freedom to enhance $Z T$, i.e., the possibility of varying the relative contributions of $\Gamma$-, $X$-, and $L$-point carriers to further enhance $Z T$ by changing the superlattice parameters such as the superlattice growth direction, superlattice period, and layer thicknesses. We emphasize that these calculations pertain to the three-dimensional (3D) figure of merit from the superlattice, denoted by $Z_{3 D} T$.

The calculation of the energy dispersion relation along the superlattice axis ( $z$ axis) was carried out at each high symmetry point ( $\Gamma, X$, and $L$ points) in the Brillouin zone using a plane-wave approximation with an appropriate boundary condition (Krönig-Penny model). ${ }^{16}$ Requiring the

\footnotetext{
${ }^{a)}$ Division of Engineering and Applied Sciences, Harvard University, Cambridge, MA 02138.

b) Also with the Department of Electrical Engineering and Computer Science. Electronic mail: millie@mgm.mit.edu
}

condition that the particle current should be conserved at the barrier-well interfaces, we apply the boundary conditions $\Psi_{\mathrm{GaAs}}=\Psi_{\mathrm{AlAs}}$ and $\left(d \Psi_{\mathrm{GaAs}} / d z\right) / m_{z(\mathrm{GaAs})}=\left(d \Psi_{\mathrm{AlAs}} / d z\right) /$ $m_{z \text { (AlAs) }}$ at the GaAs/AlAs interface, where $\Psi_{\text {GaAs }}$ and $\Psi_{\mathrm{AlAs}}$ denote the electron wave function in the GaAs and AlAs layers, respectively, and $m_{z(\mathrm{GaAs})}$ and $m_{z(\mathrm{AlAs})}$ are the $z$ components of the effective mass tensor for the GaAs and AlAs layers, respectively. The conduction-band offsets at each symmetry point are calculated using the experimentally determined empirical relation $\Delta E_{c} / \Delta E_{g}=0.68,{ }^{17}$ where $\Delta E_{c}$ is the conduction- band offset at the $\Gamma$ point, and $\Delta E_{g}$ is the difference in the direct energy-band gap at the $\Gamma$ point between GaAs and AlAs. The in-plane components of the effective-mass tensor are calculated by projecting the $3 \mathrm{D}$ constant energy surface for the well material on to the plane of the quantum wells. It is noted that for the band offsets calculated in this way the $\Gamma$ and $L$ point quantum wells, and the $X$ point quantum wells are formed within the GaAs layers and within the AlAs layers, respectively, so that we can effectively separate the carriers in different carrier pockets in real space to minimize the intervalley scattering, which is also favorable for achieving high $Z$. We tabulate the band parameters used in the present calculation from Refs. 17 and 18 in Table I .

The transport coefficients $\left(\sigma, S\right.$, and $\left.\kappa_{e}\right)$ for the whole superlattice (SL) are calculated using textbook equations in terms of the $\mathbf{L}^{(\alpha)}$ functions defined elsewhere. ${ }^{19}$ The $\mathbf{L}^{(\alpha)}$ functions for the case of a single-band two-dimensional (2D) quantum well (denoted by $\mathbf{L}_{2 \mathrm{D}}^{(\alpha)}$ ) are evaluated in Ref. 5 using the approximations of a constant relaxation time and a parabolic energy dispersion relation. For superlattice samples, which have an energy dispersion along the $z$ axis, we evaluate $\mathbf{L}_{\mathrm{SL}}^{(\alpha)}$ by

$$
\mathbf{L}_{\mathrm{SL}}^{(\alpha)}(\zeta)=\rho_{2 \mathrm{D}}^{-1} \int \mathbf{L}_{(2 \mathrm{D})}^{(\alpha)}(\zeta-\varepsilon) \frac{\partial \rho(\varepsilon)}{\partial \varepsilon} d \varepsilon,
$$

where $\rho(\varepsilon)$ is the density of states as a function of energy for the whole superlattice for the pertinent subband and $\rho_{2 \mathrm{D}}$ is the density of states for the pertinent quantum well if it had only a single subband and no band broadening $\left(\rho_{2 \mathrm{D}}\right.$ $\left.=\left(m_{t(2 \mathrm{D})} m_{l(2 \mathrm{D})}\right)^{1 / 2} / \pi d_{W} \hbar^{2}\right)$. The $\mathbf{L}_{\mathrm{SL}}^{(\alpha)}$ functions for the 
TABLE I. Band parameters at various high symmetry points for GaAs and AlAs. The offset energy denotes the energy of the bottom of the quantum wells that are formed at each symmetry point relative to the $\Gamma$ point of bulk GaAs.

\begin{tabular}{lccc}
\hline \hline Band Parameter & $\Gamma$ point & $X$ point & $L$ point \\
\hline$\left[m_{t} / m\right]_{\text {GaAs }}{ }^{a}$ & 0.067 & 0.23 & 0.0754 \\
{$\left[m_{l} / m\right]_{\text {GaAs }}{ }^{a}$} & $\ldots$ & 1.3 & 1.9 \\
{$\left[m_{t} / m\right]_{\text {AlAs }}{ }^{a}$} & 0.150 & 0.19 & 0.0964 \\
{$\left[m_{l} / m\right]_{\text {AlAs }}{ }^{\mathrm{b}}$} & $\ldots$ & 1.1 & 1.9 \\
$U_{0}(\mathrm{eV})^{\mathrm{c}}$ & 1 & 3 & 4 \\
$\mathrm{QW} \mathrm{layer}^{\mathrm{d}}$ & 1.084 & 0.242 & 0.132 \\
$\mu\left(\mathrm{cm}^{2} / \mathrm{V} \mathrm{s}\right)^{\mathrm{e}}$ & $\mathrm{GaAs}$ & $\mathrm{AlAs}$ & $\mathrm{GaAs}$ \\
Offset energy $(\mathrm{eV})^{\mathrm{a}}$ & 3000 & 180 & 950 \\
& 0 & 0.234 & 0.284 \\
\hline
\end{tabular}

${ }^{\mathrm{a}}$ Data taken from Ref. 17.

${ }^{b}$ Number of equivalent carrier pockets.

'Potential barrier height. Data taken from Ref. 17.

${ }^{\mathrm{d}}$ Material acting as quantum-well layer.

${ }^{\mathrm{e}}$ Bulk carrier mobilities for the quantum-well material at the pertinent symmetry point. Data taken from Ref. 18.

whole superlattice are calculated by summing $\mathbf{L}_{\mathrm{SL}}^{(\alpha)}$ s over all subbands, evaluated separately. We express the structure of the superlattice by denoting the GaAs and AlAs layer thicknesses $d$ in terms of $\left(d_{\mathrm{GaAs}} / d_{\mathrm{AlAs}}\right)$.

Detailed modeling for the lattice thermal conductivity has been carried out elsewhere for a GaAs/AlAs superlattice. ${ }^{20}$ Briefly, we find that the lattice thermal conductivity $\left(\kappa_{\mathrm{ph}}\right)$ for superlattices with $d_{W}=d_{B}$, where $d_{W}$ and $d_{B}$ are quantum-well and barrier-layer thicknesses, respectively, is reduced from the value for bulk GaAs $(440 \mathrm{~mW} /$ $\mathrm{cm} \mathrm{K})$ to a value of $170 \mathrm{~mW} / \mathrm{cm} \mathrm{K}$ for the $(100 \AA / 100 \AA)$ superlattice (reduction by a factor of 2.6 relative to the value for bulk GaAs), and $\kappa_{\mathrm{ph}}$ is further reduced to $73 \mathrm{~mW} / \mathrm{cm} \mathrm{K}$ for the (20 $\AA / 20 \AA)$ superlattice (factor of 6 reduction relative to bulk GaAs) at room temperature. With these data for $\kappa_{\mathrm{ph}}$ as a function of $d_{W}$, the models for other transport coefficients are combined to calculate the $Z T$ for the whole superlattice.

We first show the result of the calculation where only the $\Gamma$-point subband is assumed to be occupied (denoted as the $\Gamma$-point superlattice, hereafter). Shown in the Fig. 1 is $Z_{3 \mathrm{D}} T$ for an equal thickness $\left(d_{W}=d_{B}\right) \Gamma$-point superlattice at the optimal doping, normalized to the optimum value for the bulk $(Z T)_{\text {bulk }}$ calculated similarly. The density of states for electrons near the band edge is shown in the inset of Fig. 1 as a function of energy for the selected superlattices used in the

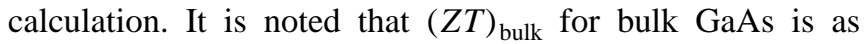
small as 0.0085 at the optimum carrier concentration, whereas $Z_{3 \mathrm{D}} T$ for the $(20 \AA / 20 \AA) \Gamma$-point superlattice is more than 0.1 at the optimum carrier concentration (see Fig. 3 ), which is more than a ten times enhancement in $Z_{3 \mathrm{D}} T$ relative to the value for bulk GaAs. The $\Gamma$-point assumption is valid for GaAs/AlAs superlattices with $d_{W}>30 \AA$ or for $\mathrm{GaAs} / \mathrm{Al}_{1-x} \mathrm{Ga}_{x}$ As superlattices with $x<0.4$.

Shown in Fig. 2(a) is the calculated density of states for all the subbands for a (001)-oriented (20 $\AA / 20 \AA)$ superlattice. By making a superlattice in this orientation, an energy splitting between the longitudinal $\left(X_{l}\right)$ and the two transverse pockets $\left(X_{t}\right)$ is introduced for the three equivalent $X$ point pockets in the $3 \mathrm{D}$ Brillouin zone. We find that the $\Gamma$,

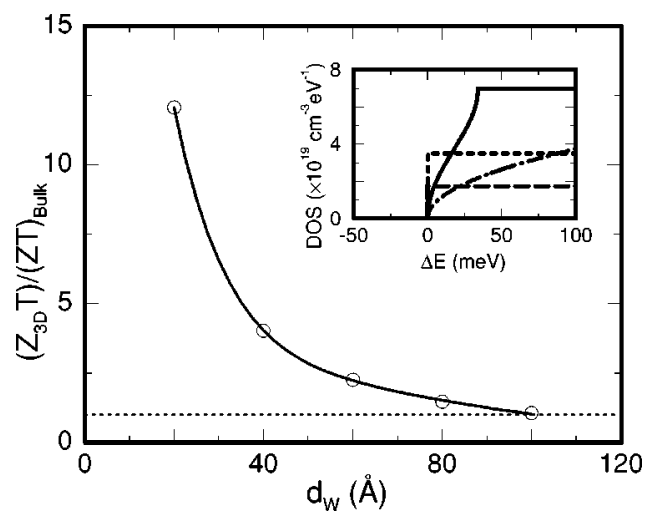

FIG. 1. Plot of the ratio of $Z_{3 \mathrm{D}} T$ for an equal thickness $\left(d_{W}=d_{B}\right)$ GaAs/ AlAs $\Gamma$-point superlattice at optimum doping concentration normalized to that of bulk GaAs calculated for $T=300 \mathrm{~K}$. The inset shows the density of states for electrons as a function of energy relative to the conduction-band edge $(\Delta E)$ for the selected superlattices $\left[d_{W}=20\right.$ (solid line), 40 (short dashed line), and 80 (long dashed line) $̊$, respectively] as well as for bulk GaAs (dash-dotted line). See Table I for the band parameters used.

$X_{t}$ and $L$ subbands lie very close in energy in this superlattice, and that the energy of $X_{l}$ is about 50-60 meV lower than that of the other subbands. Even though the mobility for the $X$-point carriers is rather low (see Table I), we nevertheless find that for all the structures considered in the present work for the (001) orientation (see below), the highest $Z_{3 \mathrm{D}} T$ occurs for the $(20 \AA / 20 \AA)$ superlattice (see Fig. 3). The results in Fig. 3 show that although at low carrier concentrations $\left(<2 \times 10^{18} \mathrm{~cm}^{-3}\right)$ the highest $Z_{3 \mathrm{D}} T$ is obtained if only the $\Gamma$-point subband is occupied, we find that $Z_{3 \mathrm{D}} T$ increases with increasing carrier concentration, and that under optimal doping conditions, $Z_{3 \mathrm{D}} T$ can be increased by occupation of other carrier pockets in the Brillouin zone when $n>2$ $\times 10^{18} \mathrm{~cm}^{-3}$. Assuming contributions from all the subbands, $Z_{3 \mathrm{D}} T$ of the superlattice is expected to peak at $Z_{3 \mathrm{D}} T \sim 0.41$ for $n \sim 6 \times 10^{19} \mathrm{~cm}^{-3}$.

It is found that the relative subband energies for the $\Gamma, X$ and $L$ carrier pockets are very sensitive to the GaAs and AlAs layer thicknesses for the superlattice. We have thus also calculated $Z_{3 \mathrm{D}} T$ for $(30 \AA / 20 \AA)$ and $(20 \AA / 30 \AA)$ su-

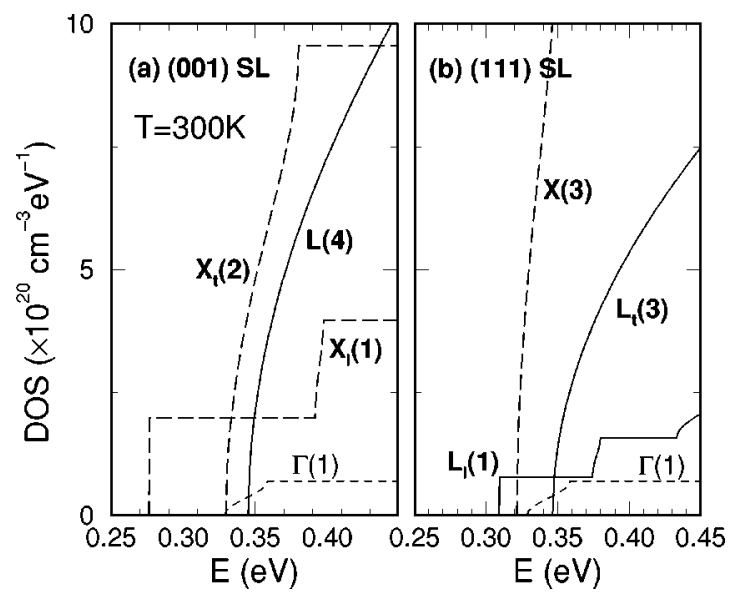

FIG. 2. Density of states for electrons in GaAs/AlAs superlattices as a function of energy relative to the $\Gamma$ point of bulk GaAs calculated at $\Gamma$ (short-dashed line), $X$ (long-dashed line), and $L$ (solid line) points: (a) a (001) - oriented GaAs $(20 \AA) / A l A s ~(20 \AA)$ superlattice and (b) a (111)oriented GaAs $(20 \AA) / \mathrm{AlAs}(20 \AA)$ superlattice. The degeneracy of each type of carrier pocket is indicated in parentheses. 


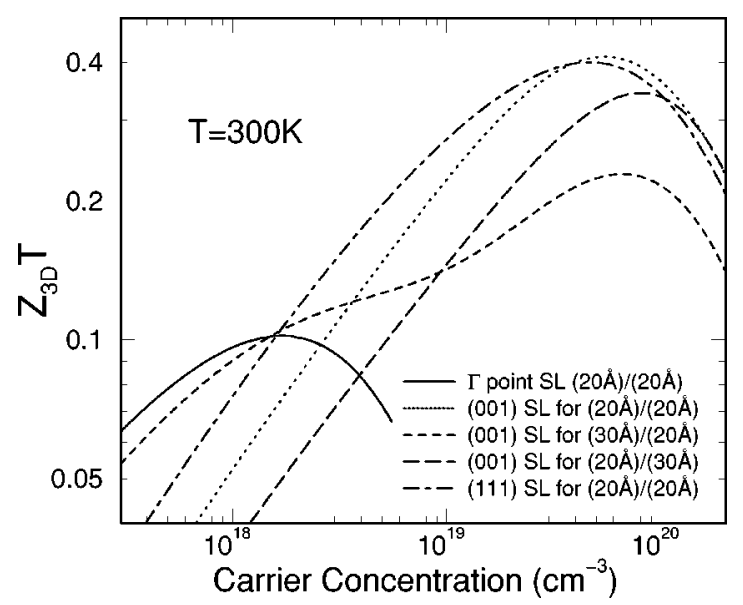

FIG. 3. Estimated $Z_{3 \mathrm{D}} T$ as a function of carrier concentration for a (001)-

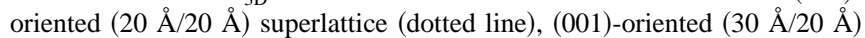
superlattice (short-dashed line), (001)-oriented (20 $\AA / 30 \AA$ ) superlattice (long-dashed line), and (111)-oriented (20 ̊/20 ̊̊) superlattice (long-dashshort-dash line). The solid line denotes $Z_{3 \mathrm{D}} T$ calculated for a $(20 \AA / 20 \AA) \Gamma$ point superlattice.

perlattices, as shown in Fig. 3. We find that the $\Gamma$ subband lies lowest in energy for a $(30 \AA / 20 \AA)$ superlattice, for which the calculated $Z_{3 \mathrm{D}} T$ is lower than that for a $(20 \AA / 20$ $\AA)$ superlattice at higher carrier concentrations $\left(>3 \times 10^{18}\right.$ $\left.\mathrm{cm}^{-3}\right)$, but higher at lower carrier concentrations $(<3$ $\left.\times 10^{18} \mathrm{~cm}^{-3}\right)$. In the case of a $(20 \AA / 30 \AA)$ superlattice (long-dashed line in Fig. 3), we find that the energy separations between the $X_{l}, X_{t}$ subbands and the $\Gamma, L$ subbands become larger compared to that for the $(20 \AA / 20 \AA)$ superlattice. Therefore, we observe a moderate decrease in $Z_{3 \mathrm{D}} T$ for this superlattice relative to the ( $20 \AA / 20 \AA)$ superlattice.

It is a crucial question whether the heavy doping condition $\left(n>10^{19} \mathrm{~cm}^{-3}\right)$ is attainable in an actual GaAs/AlAs superlattice. To address this issue, we have also explored other superlattice orientations for which a high $Z_{3 \mathrm{D}} T$ may be expected under moderate doping conditions $\left(n \sim 5 \times 10^{18}\right.$ $\mathrm{cm}^{-3}$ ). Shown in Fig. 2(b) is the calculated density of states for a (111)-oriented $(20 \AA / 20 \AA)$ superlattice. In this structure, since all the $\Gamma, X$, and $L$ subbands lie very close in energy, higher $Z_{3 \mathrm{D}} T$ s relative to the (001)-oriented superlattices are expected at lower carrier concentrations $(\sim 5$ $\times 10^{18} \mathrm{~cm}^{-3}$ ). This superlattice structure (see Fig. 3) not only yields as high a $Z_{3 \mathrm{D}} T$ value as for the (001)-oriented (20 $\AA / 20 \AA$ ) superlattice at the optimal doping level, but we can also expect a value of $Z_{3 \mathrm{D}} T \sim 0.2$ at moderate carrier concentrations $\left(\sim 5 \times 10^{18} \mathrm{~cm}^{-3}\right)$.
In summary, it is found that $Z_{3 \mathrm{D}} T$, the calculated thermoelectric figure of merit for the $\Gamma$-point superlattice could be more than ten times higher than that for bulk GaAs. Contributions from quantum wells at the $X$ and $L$ points in the Brillouin zone, can further increase $Z_{3 \mathrm{D}}$ by an additional factor of $\sim 4$. Variation of the superlattice parameters such as the superlattice period, layer thicknesses, and the superlattice growth direction to optimize $Z_{3 \mathrm{D}} T$ predicts a value of $Z_{3 \mathrm{D}} T$ $\sim 0.41$ at room temperature for either (001)- or (111)oriented superlattices under heavy doping conditions $(\sim 5$ $\left.\times 10^{19} \mathrm{~cm}^{-3}\right)$, whereas $Z_{3 \mathrm{D}} T \sim 0.2$ is expected at moderate carrier concentrations $\left(\sim 5 \times 10^{18} \mathrm{~cm}^{-3}\right)$ for a (111)-oriented $(20 \AA / 20 \AA)$ superlattice. These values for $Z_{3 \mathrm{D}} T$ are still much greater than the $Z T$ s for bulk GaAs $(Z T \sim 0.0085)$ or for the $\Gamma$-point superlattice $\left(Z_{3 \mathrm{D}} T \sim 0.1\right)$ at $300 \mathrm{~K}$ obtained using the same basic models.

The authors would like to thank Dr. G. Chen and Dr. G. Dresselhaus for valuable discussions. The authors gratefully acknowledge support from the Honda Corporation and from ONR under MURI Subcontract No. 205-G-7A114-01.

${ }^{1}$ L. D. Hicks and M. S. Dresselhaus, Phys. Rev. B 47, 12727 (1993).

${ }^{2}$ L. D. Hicks and M. S. Dresselhaus, Phys. Rev. B 47, 16631 (1993).

${ }^{3}$ L. D. Hicks, T. C. Harman, X. Sun, and M. S. Dresselhaus, Phys. Rev. B 53, R10493 (1996).

${ }^{4}$ T. C. Harman, D. L. Spears, and M. J. Manfra, J. Electron. Mater. 25, 1121 (1996).

${ }^{5}$ Lyndon D. Hicks, Ph.D. thesis, Massachusetts Institute of Technology, 1996.

${ }^{6}$ T. C. Harman, D. L. Spears, D. R. Calawa, S. H. Groves, and M. P. Walsh, Proceedings of the16th International Conference on Thermoelectrics (1997), p. 416.

${ }^{7}$ J. O. Sofo and G. D. Mahan, Appl. Phys. Lett. 65, 2690 (1994).

${ }^{8}$ D. A. Broido and T. L. Reinecke, Appl. Phys. Lett. 67, 100 (1995).

${ }^{9}$ D. A. Broido and T. L. Reinecke, Appl. Phys. Lett. 67, 1170 (1995).

${ }^{10}$ P. J. Lin-Chung and T. L. Reinecke, Phys. Rev. B 51, 13244 (1995).

${ }^{11}$ D. L. Broido and T. L. Reinecke, Phys. Rev. B 51, 13797 (1995).

${ }^{12}$ D. A. Broido and T. L. Reinecke, Appl. Phys. Lett. 70, 2834 (1997).

${ }^{13}$ D. M. Rowe and G. Min, Proceedings of the 13th International Conference on Thermoelectrics (1994), p. 339.

${ }^{14}$ Y. Nishio and T. Hirano, Jpn. J. Appl. Phys., Part 2 36, 170 (1997).

${ }^{15}$ R. Venkatasubramanian, T. Colpitts, E. Watko, M. Lamvik, and N. ElMasry, J. Cryst. Growth 170, 817 (1997).

${ }^{16}$ C. Kittel, Introduction to Solid State Physics, 7th ed. (Wiley, New York, 1996), p. 180.

${ }^{17}$ S. Adachi, J. Appl. Phys. 58, R1 (1985).

${ }^{18}$ Numerical Data and Functional Relationships in Science and Technology, New Series, Vol. 22a, Landolt-Bornstein (Springer, Berlin, 1987).

${ }^{19}$ N. W. Ashcroft and N. D. Mermin, Solid State Physics (W. B. Saunders, Philadelphia, PA, 1976), Chaps. 12 and 13.

${ }^{20}$ G. Chen, Trans. ASME, Ser. C: J. Heat Transfer 119, 220 (1997). 